\title{
LVIII. On the coefficients of self and mutual induction of coaxial coils
}

\section{S. Butterworth M.Sc.}

To cite this article: S. Butterworth M.Sc. (1915) LVIII. On the coefficients of self and mutual induction of coaxial coils, Philosophical Magazine Series 6, 29:172, 578-592, DOI: 10.1080/14786440408635335

To link to this article: http://dx.doi.org/10.1080/14786440408635335

曲 Published online: 08 Apr 2009.

Submit your article to this journal $\pi$

Џ Article views: 11

Q View related articles $\square$

4 Citing articles: 4 View citing articles ๘ 
LVIII. On the Coefficients of Self and Mutual Induction of Coaxial Coils. By S. ButTenworth, M.Sc., Lecturer in Physics, School of Technology, Manchester * .

1. ATHOUGH many formulæ have been given for the A mutual induction of coaxial circles and solenoids, little seems to have been done on the mutual induction of coils for which the ratio of the inner and outer diameters differs considerably from unity. The present investigation is to supply suitable formulæ for such cases.

The method adopted is to find the mutual induction between two mutually external semi-infinite coaxial coils having zero core diameters and unit winding density (i.e. the number of turns per unit area of channel section is unity), and then by applying the laws of combination of mutual inductances to find the mutual induction between finite hollow coils.

The results are extended so as to include self-induction.

The semi-infinite coils of the nature indicated will, for brevity, be referred to as "solid coils." If we take the radius of the larger coil as the unit of length, then only two variables are involved, viz. : the radius of the smaller coil ranging from zero to unity and the distance of the coil faces. Dimensional considerations will give the correct formula when the radius of the larger coil is not unity.

2. For any magnetic field possessing circular symmetry about an axis, the magnetic potential $\boldsymbol{\Omega}$ satisfies the equation

$$
\frac{\partial^{2} \Omega}{\partial \rho^{2}}+\frac{1}{\rho} \frac{\partial \Omega}{\partial \rho}+\frac{\partial^{2} \Omega}{\partial z^{2}}=0, . . . . . .
$$

in which $z$ represents the distance along, and $\rho$ the distance from the axis of symmetry. A solution of this equation is

$$
\Omega=\int_{0}^{\infty} \phi(\lambda) e^{-\lambda z} J_{0}(\lambda \rho) d \lambda \text {. . . . . }
$$

reducing when $\rho=0$ to

$$
\Omega_{0}=\int_{0}^{\infty} \phi(\lambda) e^{-\lambda z} d \lambda . . . . . . .
$$

If $\Phi$ is the stream function (i.e. the magnetic flux through

* Communicated by the Author. 
a circle of radius $\rho$ and centre at $\approx, 0$ ), then

$$
\begin{aligned}
\Phi & =-2 \pi \int_{0}^{\rho} \rho \frac{\partial \Omega}{\partial z} d \rho \\
& =2 \pi \rho \int_{0}^{\infty} \phi(\lambda) e^{-\lambda z} \mathrm{~J}_{1}(\lambda \rho) d \lambda ; . . . \quad .
\end{aligned}
$$

or on expanding $J_{1}(\lambda \rho)$ in ascending powers of $\rho$ and making use of (3),

$$
\Phi=-\pi \rho^{2} \sum_{0}^{\infty}(-)^{n}\left(\frac{\rho}{2}\right)^{2 n} \frac{1}{i n+1} \frac{d^{2 n+1} \Omega_{0}}{d z^{2 n+1}} .
$$

so that $\Phi$ can be found at all points if the potential at all points along the axis of symmetry is given.

Now let a solid coil of radius $r$ extending from $z$ to infinity be placed with its axis along the axis of symmetry. The number of linkages that the field makes with this coil is

$$
\begin{aligned}
\mathrm{N} & =\int_{0}^{r} d \rho \int_{z}^{\infty} \Phi d z \\
& =\pi r^{3} \sum_{0}^{\infty} \frac{(-)^{n}}{2^{2 n}} \frac{r^{2 n}}{(2 n+3) \underline{\ln } \underline{\ln +1} \frac{d^{2 n} \Omega_{0}}{d z^{2 n}} . . .}
\end{aligned}
$$

3. If $\Omega_{0}$ is due to a second solid coil of unit radius exteuding from $\approx=0$ to $:=-\infty$, then

$$
\begin{aligned}
\Omega_{0} & =2 \pi \int_{0}^{2}\left(\sqrt{\rho^{2}+z^{2}}-\rho\right) d \rho \\
& =\pi\left(z^{2} \log \frac{1+\zeta}{z}+\zeta-2 z\right), \ldots . .
\end{aligned}
$$

where $\zeta^{2}=1+z^{2}$.

If $z$ is large then (7) may be expanded in inverse powers of $z$ giving

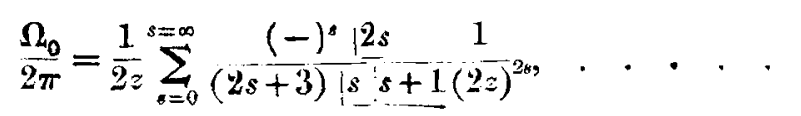

so that

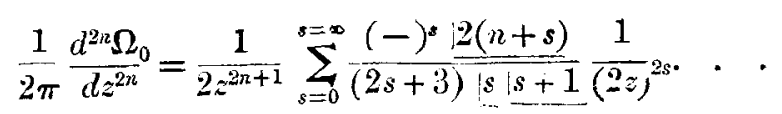


Inserting (9) in (6) and rearranging so as to express $N$ in inverse powers of $z$,

$$
\frac{\mathrm{N} z}{\pi^{2} r^{3}}=\sum_{p=0}^{p=\infty} \frac{(-)^{p} \mid 2 p}{(2 z)^{2 p}} \sum_{n=0}^{n=p}(2 n+3)(2 p-2 n+3) \underline{|n| p-n} r^{2 n} n+1 \mid p-n+1
$$

or on expansion

$$
\begin{aligned}
\frac{\mathrm{N} z}{\pi^{2} r^{3}}=\frac{1}{9} & -\frac{1}{(2 z)^{2}}\left(\frac{1}{15}+\frac{r^{2}}{15}\right)+\frac{1}{(2 z)^{4}}\left(\frac{2}{21}+\frac{6}{25} r^{2}+\frac{2}{21} r^{4}\right) \\
& -\frac{1}{(2 z)^{6}}\left(\frac{5}{27}+\frac{6}{7} r^{2}+\frac{6}{7} r^{4}+\frac{5}{27} r^{6}\right) \\
& +\frac{1}{(2 z)^{8}}\left(\frac{14}{33}+\frac{28}{9} r^{2}+\frac{40}{7} r^{4}+\frac{28}{9} r^{6}+\frac{14}{33} r^{8}\right)
\end{aligned}
$$

From this formula, $\mathrm{N}$ (which represents the mutual induction between the two solid coils) can be obtained to 1 in 100,000 if $z>3$.

4 . When $z$ is small (7) may be expanded in direct powers of $z$ giving

$$
\begin{aligned}
\frac{\Omega_{0}}{2 \pi}=\frac{1}{2}-z+\frac{1}{2} z^{2} & \left(\left(\log \frac{2}{z}+\frac{1}{2}\right)\right. \\
& +\sum_{s=2}^{s=\infty} \frac{(-)^{s}}{2^{2 s-1}} \frac{2 s-3}{\sqrt{s-1}} z^{2 s} . . . . .
\end{aligned}
$$

In this formula the factor $-\frac{1}{2} z^{2} \log z$ requires special treatment. Denoting it by $\omega / 2 \pi$,

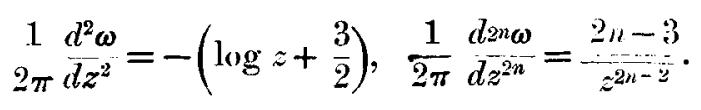

Hence by (6), if $n$ represents the number of linkages due to $\omega$,

$$
\begin{aligned}
\frac{\therefore}{2 \pi^{2} r^{3}}=-\frac{1}{3} z^{2} & \log z+\frac{p^{2}}{40}\left(\log z+\frac{3}{2}\right) \\
& +z^{2} \sum_{n=2}^{n=\infty} \frac{(-)^{n} \frac{2 n-3}{(2 n+3)}\left(\frac{r}{2 z}\right)^{2 n}}{n} . . .
\end{aligned}
$$

This converges so long as $z>r$.

For the remaining portion of $\Omega_{0}$ it is preferable to retain the finite form (7) because of the slow convergence of (11). 
Self and Mlutual Induction of Coadical coils.

Hence as a working formula for $z>r$ we have

$$
\underset{2 \pi^{2} r^{3}}{\mathrm{~N}}=\mathrm{A}-(\mathrm{B}-\mathrm{a}) r^{2}+\left(\mathrm{i} r^{4}-\mathrm{D} r^{6} . .\right.
$$

in which

$$
\left.\begin{array}{rl}
3 \mathrm{~A} & =\frac{1}{2} z^{2} \log \frac{1+\zeta}{z}+\frac{1}{2} \zeta-z \equiv \frac{\Omega_{0}}{2 \pi} \\
40 \mathrm{~B} & =\log \frac{1+\zeta}{z}-\frac{1}{\zeta} \equiv \frac{1}{2 \pi} \cdot \frac{d^{2} \Omega_{0}}{d z^{2}} \\
1344 \mathrm{C} & =-\frac{1}{\zeta}\left(\frac{1}{1+\zeta}+\frac{1}{\zeta^{2}}-\frac{3}{\zeta^{4}}\right) \\
82,944 \mathrm{D} & =\frac{3}{\zeta}\left(\frac{1}{(1+\zeta)^{2}}-\frac{1}{\zeta^{2}}-\frac{3}{\zeta^{2}}+\frac{35}{\zeta^{6}}-\frac{35}{\zeta^{8}}\right) \\
\alpha & =\left(\frac{z}{n}\right)^{2} \sum_{n=2}^{n=x} \frac{(-)^{n}}{(2 n+3)} \frac{2 n-3}{n ! n+1}\left(\frac{y^{\prime}}{2 z}\right)^{2 n}
\end{array}\right\}
$$

If we use the series formula (8) and (9) for $3 \mathrm{~A}$ and $40 \mathrm{~B}$, when $z>2$ the range of formula (B) is $4>z>r$.

5 . When $z$ is less than $r$, the method followed in the preceding sections fails to give in convergent series, because of the logarithmic term in (11). For this case the method adopted is to find some simple magnetic distribution which will give riso to an axial potential containing the term $\pi \tau^{2} \log z$ and other terms for which the preceding method is applicable. The linkages due to this distribution are calculated by direct integration, while those due to the terms not containing $\log z$ are found by the preceding method. The difference between the two results gives the linkages due to $\pi z^{2} \log z$.

Let there be a linear distribution of poles on the axis of $z$ extending from $z=0$ to $z=-c$, and having a density $\pi t^{2}$. The potential at $z$ dne to this distribution is

$$
\begin{aligned}
\omega^{\prime} & =\pi \int_{z}^{c+z} \frac{(x-z)^{2}}{u} d x \\
& =\omega+\pi\left\{z^{2} \log (c+z)-2 c z+\frac{c^{2}}{2}\right\}, . .
\end{aligned}
$$

in which as before $\omega=-\pi z^{2} \log z$.

Since we only require the linkages $n$ corresponding to $\omega$, we can choose $c$ to have any convenient value. It will be supposed that $c$ is very large. Then, by the method of 
Section 2, if $n^{\prime}$ are the linkages corresponding to $\omega^{\prime}$,

$$
\begin{aligned}
\frac{n^{\prime}-n}{2 \pi^{2} r^{3}} & =\frac{1}{3}\left\{\frac{1}{2} z^{2} \log (c+z)-c z+\frac{c^{2}}{4}\right\}-\frac{r^{2}}{40} \log (c+z) \\
& + \text { terms which vanish when } \sigma \text { is infinite. }
\end{aligned}
$$

To obtain the linkages $n^{\prime}$ by direct integration we must find the work to be done to remove the linear magnetic distribution from the field due to the solid coil of radius $r$ whose (south-seeking) pole is at $z, 0$.

Now the axial potential at a distance $x$ from this pole is by (7)

$$
-\Omega_{0}(x, r)=-\pi\left(x^{2} \log \frac{r+\sqrt{r^{2}+x^{2}}}{r}+r \sqrt{r^{2}+x^{2}}-2 r^{2} x\right),(
$$

and

$$
n^{\prime}=-\pi \int_{z}^{c+z}(x-z)^{2} \Omega_{0}(x, v) d x \quad . \quad .
$$

By direct integration,

$$
\begin{aligned}
\frac{1}{\pi} \int^{x} & (x-z)^{2} \Omega_{0}(x, r) d x \\
= & \left(\frac{1}{5} x^{2}-\frac{1}{2} x^{4} z+\frac{1}{3} x^{3} z^{2}\right) \log \frac{r+\sqrt{r^{2}+x^{2}}}{x} \\
& -\left(\frac{1}{20} r^{5}-\frac{1}{3} r^{3} z^{2}\right) \log \frac{x+\sqrt{r^{2}+r^{2}}}{r} \\
& +r \sqrt{r^{2}+x^{2}}\left(\frac{3}{10} x^{3}+\frac{1}{20} x r^{2}-\frac{5}{6} x^{2} z-\frac{1}{3} r^{2} z+\frac{2}{3} x z^{2}\right) \\
& -2 r\left(u^{4}-\frac{2}{3} x^{3} z+\frac{1}{2} x^{2} z^{2}\right) \cdot . \cdot . \cdot . \cdot \cdot(17) \\
= & \left.-\frac{1}{3} r^{4} z \text { (when } i=0\right) .
\end{aligned}
$$

Putting,$=c+z$, and supposing $c$ linge, (17) becomes

$$
\begin{aligned}
& \text {;: }\left\{2 z^{2} \log \frac{2(r+z)}{r}-\frac{4}{3} z^{2}-2 c z+c^{2}\right\} \\
& -\frac{r^{5}}{20}\left\{\log \frac{2(r+z)}{r^{2}}-\frac{1}{20}\right\} \\
& + \text { terms which vanish when } c \text { is infinite. . }
\end{aligned}
$$

When $x$ is small, the integration (17) can be obtained in series by using the form (11) for $\Omega_{0}$, and integrating term by term, the constant of integration being $-1, r^{4} z$ to correspond with (18). 
Self and Mutual Induction of Coaxial Coils.

Using this method for the lower limit of (16), and combining with (14),

$$
\begin{aligned}
& \frac{n}{2 \pi^{2} r^{3}}=\frac{1}{3}\left(\frac{1}{2} z^{2} \log \frac{2}{r}-\frac{1}{3} z^{2}\right)-\frac{r^{2}}{40}\left(\log \frac{2}{r}-\frac{1}{20}\right) \\
& +\frac{r^{2}}{2}\left\{\frac{1}{3} \frac{z}{r}-\frac{1}{3} \frac{z^{3}}{r^{3}}+\frac{1}{6} \frac{z^{4}}{r^{4}}-\frac{1}{30} \frac{z^{5}}{r^{5}}\left(\log \frac{2 r}{z}+\frac{77}{60}\right)\right. \\
& \left.-\frac{z^{3}{ }^{3}=\infty}{r^{3}} \sum_{n=2} \frac{(-)^{n}}{2^{2 n-2}} \frac{\mid 2 n-3}{n-1} \frac{z^{2 n} / r^{2 n}}{(2 n+1)(2 n+3)}\right\} \text {. }
\end{aligned}
$$

Using this to replace (12), we have instead of (B),

$$
\frac{\mathrm{N}}{2 \pi^{2} r^{3}}=\left(\frac{z^{2}}{6}-\frac{r^{2}}{40}\right) \log \frac{1}{r}+A^{\prime}-\left(B^{\prime}-\beta\right) r^{2}+C r^{4}-D r^{6} \text {. }
$$

in which

$$
\left.\begin{array}{rl}
3 \mathrm{~A}^{\prime} & =\frac{1}{2} z^{2} \log 2(1+\zeta)+\frac{1}{2} \zeta-z-\frac{1}{3} z^{2}, \\
40 \mathrm{~B}^{\prime} & =\log 2(1+\zeta)-\frac{1}{\zeta}+\frac{29}{20}, \\
\beta= & \frac{2}{2 r}\left\{\frac{1}{3}-\frac{1}{3} \frac{z^{2}}{r^{2}}+\frac{1}{6} \frac{z^{3}}{r^{3}}-\frac{1}{30} \frac{z^{4}}{r^{4}}\left(\log \frac{2 r}{z}+\frac{77}{60}\right)\right. \\
& \left.\quad-\frac{z^{2}{ }^{2}=\infty}{r^{2}} \sum_{n=2} \frac{(-)^{n}}{2^{2 n-2}} \frac{2 n-3}{n-1} \ln \frac{z^{2 n} / r^{2 n}}{(2 n+1)(2 n+3)}\right\}
\end{array}\right\}
$$

6. When $z=0$, (19) becomes

$$
\frac{n}{2 \pi^{2} r^{3}}=-\frac{r^{2}}{40}\left(\log _{r^{2}} \frac{2}{20}\right) . \quad \text {. . . }
$$

Hence by (6) and (11),

$$
\frac{\mathrm{N}}{2 \pi^{2} r^{3}}=\frac{1}{6}-\frac{r^{2}}{40}\left(\log \frac{4}{r}+\frac{9}{20}\right)+\frac{1}{2} r^{4} \sigma
$$

where

$$
\begin{aligned}
\sigma & =\sum_{n=2}^{n=\infty} \frac{|2 n-3| 2 n}{2^{4 n-2}(2 n+3)|n| \frac{r^{2 n-4}}{|n| n-1} \mid \underline{n+1}} \\
& =\sum_{0}^{\infty}\left(\frac{1.3 .5 \ldots \ldots 2 n+3}{2.4 .6 \ldots \ldots 2 n+4}\right)^{2} \frac{r^{2 n}}{(2 n+7)(2 n+3)(n+3)(n+1)}
\end{aligned}
$$

7. As a check on this result, the case of $z=0$ will now be treated by another method, involving elliptic integrals. The method is to start with the known elliptic integral formuli for the mutual induction between two coaxial solenoids and, 
by integration, to find the mutual induction between two solid coils.

Certain reduction formulæ are required, and these will be dealt with first. If $E$ and $K$ are complete elliptic integrals of the first and second kind to modulus $x$, then

$$
\frac{d \mathrm{E}}{d x}=\frac{\mathrm{E}-\mathrm{K}}{x}, \quad \frac{d \mathrm{~K}}{d x}=\frac{\mathrm{E}}{x\left(1-x^{2}\right)}-\frac{\mathrm{K}}{x} . .
$$

From (21) we readily derive the following reduction formulæ,

$$
\begin{gathered}
(n+2) \int x^{n} \mathrm{E} d x=x^{n+1} \mathrm{E}+\int x^{n} \mathrm{~K} d x \\
(n+2)^{2} \int x^{n+2} \mathrm{~K} d x=x^{n+1} \mathrm{E}-(n+2) x^{n+1}\left(1-x^{2}\right) \mathrm{K} \\
\quad+(n+1)^{2} \int x^{n} \mathrm{~K} d x \ldots \\
\int \frac{\mathrm{E}}{x^{2}} d x=\left(\frac{1}{x}-x\right) \mathrm{K}-2 \frac{\mathrm{E}}{x}, \quad . \quad . \quad .
\end{gathered}
$$

by meaus of which $\int x^{n} \mathrm{E} d x, \int x^{n} \mathrm{~K} d x$ can be expressed in terms of $\mathrm{E}, \mathrm{K}, \int \mathrm{K} d x$ and $\int \frac{\mathrm{K}}{x}$, if $n$ is any integer positive or negative.

In the succeeding work we are particularly concerned with

$$
\begin{aligned}
& u=\int_{0}^{x} \mathrm{~K} d x \text {, and } v=\int_{x}^{1} \frac{\mathrm{K}}{x} d x . \\
& \text { Expressing } \mathrm{K} \text { in series, } \\
& u=\frac{\pi}{2} x\left\{1+\sum_{1}^{\infty}\left(\frac{1.3 .5 \ldots \ldots 2 n-1}{2.4 .6 \ldots \ldots 2 n}\right)^{2} \frac{x^{2 n}}{2 n+1}\right\} .
\end{aligned}
$$

When $x=1$,

$$
\begin{aligned}
u=u_{1} & =\int_{0}^{\frac{\pi}{2}} d \theta \int_{0}^{1} \frac{d x}{\left(1-x^{2} \sin ^{2} \theta\right)^{\frac{1}{2}}}=\int_{0}^{\frac{\pi}{2}} \frac{\theta d \theta}{\sin \theta} \\
& =2\left(1-\frac{1}{3^{2}}+\frac{1}{5^{2}}-\ldots\right)=1.83193 . . .
\end{aligned}
$$


Also

$$
\begin{aligned}
v & =\int_{0}^{\frac{\pi}{2}} d \theta \int_{x}^{1} \frac{d x}{x\left(1-x^{2} \sin ^{2} \theta\right)^{\frac{1}{2}}}=\int_{0}^{\pi} d \theta \int_{x \sin \theta}^{\sin \theta} \frac{d y}{y\left(1-y^{2}\right)^{\frac{1}{2}}} \\
& =\frac{\pi}{2} \int_{x}^{1} \frac{d y}{y\left(1-y^{2}\right)^{\frac{1}{2}}}-\int_{0}^{\frac{\pi}{2}} \frac{\theta d \theta}{\sin \theta}+\int_{0}^{\frac{\pi}{2}} \frac{\theta \cot \theta d \theta}{\left(1-x^{2} \sin ^{2} \theta\right)^{\frac{1}{2}}} .
\end{aligned}
$$

by integralion by parts.

Performing the last integral of (28) by expanding $\left(1-x^{2} \sin ^{2} \theta\right)^{-\frac{3}{2}}$ in ascending powers of $x$, and integrating term by term,

$$
v+u_{1}=\frac{\pi}{2}\left\{\log \frac{4}{x}-\sum_{1}^{\infty}\left(\frac{1.3 \cdot 5 \ldots \ldots 2 n-1}{2.4 .6 \ldots \ldots 2 n}\right)^{2} \frac{x^{2 n}}{2 n}\right\} .
$$

8. The mutual induction between two semi-infinite coaxial cylinders of radii $a$ and $b$, external to each other and with their ends in contact, is

$$
m=\frac{8}{3} \pi a\left\{\left(a^{2}+b^{2}\right) \mathrm{E}-\left(a^{2}-b^{2}\right) \mathrm{K}\right\}, \quad .
$$

the linear winding density being unity, and the molulus of $\mathrm{E}$ and $\mathrm{K}$ being $b / a$, with $a>b$.

The mutual induction between a solenoid of radius $a$ and a solid coil of radius $b(a>b)$ with their ends in contact is

$$
\left.m_{1}=\frac{8}{3} \pi a \int_{0}^{b} ;\left(a^{2}+b^{2}\right) \mathrm{E}-\left(a^{2}-b^{2}\right) \mathrm{K}\right\} a b, \quad \text {. }
$$

which on applying the reduction formula becomes

$$
m_{1}=\frac{1}{3} \pi a^{4}\left\{\left(\frac{13}{2}+2 \frac{b^{2}}{a^{2}}\right) \frac{b}{a} \mathrm{E}-5\left(1-\frac{b^{2}}{a^{2}}\right) \frac{b}{a} \mathrm{~K}-\frac{3}{2} u\right\} .
$$

When $b=a$,

$$
m_{1}=m_{2}=\frac{1}{3} \pi b^{4}\left(\frac{17}{2}-\frac{3}{2} u_{1}\right) . \quad \text {. . . }
$$

The mutual induction between a solenoid of radius $b$ and a solid coil of radius $a(a>b)$ with their ends in contact is

$$
\begin{aligned}
m_{3}= & m_{2}+\int_{b}^{a} m d a \\
= & \frac{1}{3} \pi a^{4}\left\{\left(2+\frac{13}{2} \frac{b^{2}}{a^{2}}\right) \mathrm{E}-\frac{1}{2}\left(4-\frac{b^{2}}{a^{2}}-3 \frac{b^{4}}{a^{4}}\right) \mathrm{K}\right. \\
& \left.-\frac{3}{2} \frac{b^{4}}{a^{4}}\left(v+u_{1}\right)\right\} .
\end{aligned}
$$


Finally, the mutual induction between two solid coils of radii $a$ and $b$ with their ends in contact is

$$
\begin{aligned}
\mathrm{N} & =\int_{0}^{b} m_{\mathbf{3}} d b \\
& =\frac{\pi a^{5}}{30}\left\{17 r\left(1+r^{2}\right) \mathbf{E}-r\left(14+3 r^{2}\right)\left(1-r^{2}\right) \mathbf{K}\right. \\
& \left.\quad-3 \imath-3 r^{5}\left(v+u_{1}\right)\right\} . .
\end{aligned}
$$

in which $r=b / \alpha$.

When $r=1$,

$$
\mathrm{N}=\frac{\pi a^{5}}{15}\left(17-3 u_{1}\right)=2 \cdot 4094 a^{5}(\text { by }(27)) . .
$$

Inserting the series for $\mathrm{E}, \mathrm{K}, u, v$ in (35) we find

where

$$
\frac{\mathrm{N}}{2 \pi^{2} a^{5}}=\frac{r^{3}}{6}\left\{1-\frac{3}{20} r^{2}\left(\log \frac{4}{r}+\frac{9}{20}\right)+3 r^{4} \sigma\right\},
$$

$$
\sigma=\sum_{0}^{\infty}\left(\frac{1.3 .5 \ldots 2 n+3}{2.4 .6 \ldots 2 n+4}\right)^{2} \frac{r^{2 n}}{(2 n+7)(2 n+3)(n+3)(n+1)}
$$

This result is identical with formula (1)).

When $r=1$, we find (to five figures)

$$
\mathrm{N}=2 \cdot 4094 a^{\mathrm{s}} \text {, }
$$

which is in agreement with (36).

9. By means of formula (A), (B), (C), and (D) it is possible to evaluate $\mathrm{N}$ for all values of $z$, and all values of $r$ up to unity.

The formulæ have the following ranges :-

$$
\begin{array}{ll}
\text { (A) } z>3, & \text { (B) } 4>z>r, \\
\text { (C) } r>z>0, & \text { (D) } z=0 .
\end{array}
$$

Table I. shows the agreement of (A) and (B) when $z=4$; Table II. shows the agreement of (B) and (C) when $z=r$; $\frac{\mathrm{N}}{2 \pi^{2} r^{3}}$ being the numbers tabulated.

In Table III. are tabulated $\xi=\frac{N}{2 \pi^{2} r^{3}}$, for $z<4$, and in Table IV. are $\eta=\frac{1}{9}-\frac{\mathrm{N} z}{\pi^{2} r^{3}}$, the forms $\xi$ and $\eta$ being chosen as being the most suitable for graphical interpolation. For interpolation in $\boldsymbol{\eta}$ it is convenient to notice that $\eta$ is almost linear in $r^{2}$ and in $1 / z^{2}$. 
Self and Mutual Induction of Coarial Coils.

TabLe I. $z=4$.

\begin{tabular}{|c|c|c|c|c|c|}
\hline$r=$ & 0.2 . & 0.4 . & 06. & 0.8 & 1.0. \\
\hline Furmula (A & 0.0137566 & $0 \cdot 0137+19$ & 0.0137179 & 0.0136836 & 0.0136406 \\
\hline$\{\quad, \quad(\mathrm{B}$ & 0.0137564 & $0 \cdot 0137416$ & 0.0137172 & 0.0136834 & 0.0136405 \\
\hline
\end{tabular}

TABLE II.

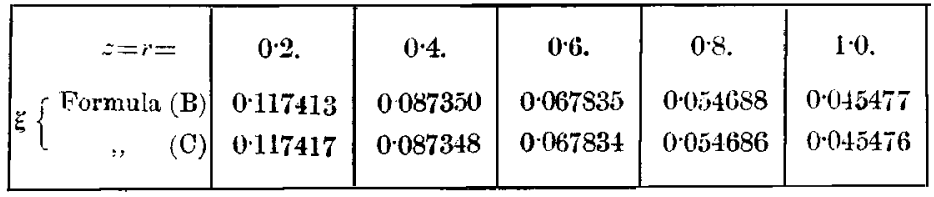

TABLE III. $\xi=\frac{\mathrm{N}}{2 \pi^{2} r^{3}}$.

\begin{tabular}{|c|c|c|c|c|}
\hline$r$ & $z=0$. & 0.2. & 0.4. & 06. \\
\hline 1.0 & $0 \cdot 122063$ & 0.09469 & $0 \cdot 075971$ & 0.062649 \\
\hline 0.8 & $0 \cdot 134215$ & $0 \cdot 10182$ & 0.080307 & 0.065405 \\
\hline 06 & $0 \cdot 145694$ & $0 \cdot 10835$ & $0.08+106$ & 0.067835 \\
\hline 04 & $0 \cdot 155685$ & 0.11378 & 0.087350 & $0 \cdot 069746$ \\
\hline 02 & $0 \cdot 163223$ & $0 \cdot 117+1$ & 0089389 & 0.070970 \\
\hline$r$. & $z=0.8$. & 10. & $2 \cdot 0$ & 4.0 \\
\hline $1 \cdot 0$ & 0.052866 & 0.045477 & 0.026022 & 0.013641 \\
\hline $0 \cdot 8$ & 0.054687 & 0.046722 & $0026: 297$ & 0.013684 \\
\hline $0 \cdot 6$ & 0.056260 & 0.047778 & 0.026520 & 0.013717 \\
\hline $0 \cdot 4$ & 0.057477 & 0.048585 & 0.026685 & $0.0137 \pm 2$ \\
\hline $0 \cdot 2$ & 0.058249 & 0.049093 & 0.026785 & 001350 \\
\hline
\end{tabular}

TABLI IV. $\quad \eta=\frac{1}{9}-\frac{\mathrm{N} z}{\pi^{2} r^{3}}$.

\begin{tabular}{|c|c|c|c|c|c|}
\hline$\therefore$ & $z=4$. & 5. & 6. & 8. & 10. \\
\hline $1 \cdot 0$ & $0 \cdot 001986$ & 1292 & 0906 & 0514 & 0331 \\
$0 \cdot 8$ & 1642 & 1066 & 0746 & 0423 & 0271 \\
06 & 1373 & 0888 & 0620 & 0351 & 0225 \\
0.4 & 1176 & 0760 & 0530 & 0300 & 0192 \\
0.3 & 1058 & 0683 & 0476 & 0269 & 0173 \\
\hline
\end{tabular}


10. Mutual inductances of finite coils.

(a) Non-overlapping coils.

By definition, $\mathrm{N}$ gives the mutual induction between two semi-infinite solid coils of unit winding density, having radii $r$ and unity, the distance of their faces being $\approx$. If the radii of the coils are $a$ and $b$, the separation $c$, and the winding densities $n_{1}, n_{2}$, then by dimensions the mutual induction is

with $b<a$.

$$
n_{1} n_{2} a^{5} \mathrm{~N}\left(\frac{v}{a}, \frac{b}{a}\right) \cdot \ldots . . .
$$

If $b>a$, then from the reciprocal property

$$
a^{5} \mathrm{~N}\left(\frac{c}{a}, \frac{b}{a}\right)=b^{5} \mathrm{~N}\left(\frac{c}{b}, \frac{a}{b}\right) . . . .
$$

If the coils are hollow, and the imer and outer radii are $a_{1}, a_{2} ; b_{1}, b_{2}$ respectively, then from the laws of combination of mutual inductances, the mutual induction (M) is given by

$$
\begin{aligned}
\mathrm{M} / n_{1} u_{2}=a_{2}^{5} & \left\{\mathrm{~N}\left(\frac{c}{a_{2}}, \frac{b_{2}}{a_{2}}\right)-\mathrm{N}\left(\frac{c}{a_{2}}, \frac{b_{1}}{a_{2}}\right)\right\} \\
& -a_{1}^{5}\left\{\mathrm{~N}\left(\frac{c}{a_{1}}, \frac{b_{2}}{a_{1}}\right)-\mathrm{N}\left(\frac{c}{a_{1}}, \frac{b_{1}}{a_{1}}\right)\right\} . .
\end{aligned}
$$

When the coils have the same radii (41) becomes (nsing (40))

$\mathrm{N} / n_{1} n_{2}=a_{2}{ }^{5} \mathrm{~N}\left(\frac{c}{a_{2}}, 1\right)-2 a_{2}^{5} \mathrm{~N}\left(\frac{c}{a_{2}}, \frac{a_{1}}{a_{2}}\right)+a_{1}^{5} \mathrm{~N}\left(\frac{c}{a_{1}}, 1\right)$.

If in addition, the coils are in contact

$$
\mathrm{M} / n_{1} n_{2}=\left(a_{2}^{5}+a_{1}^{5}\right) \mathrm{N}(0,1)-2 a_{2}^{5} \mathrm{~N}\left(0, \frac{a_{1}}{a_{2}}\right) .
$$

Now let the coils be finite and of lengths $2 l_{1}, 2 l_{2}$, the distance of their mid-points being $h$. From the laws of combination of mutual inductances we find

$$
\begin{aligned}
\mathbf{M}=\mathbf{M} & \left(l_{l}-l_{1}-l_{2}\right)+\mathbf{M}\left(h+l_{1}+l_{2}\right) \\
& \quad-\mathbf{M}\left(l-l_{1}+l_{2}\right)-\mathbf{M}\left(h+l_{1}-l_{2}\right), \quad . \quad .
\end{aligned}
$$

in which $\mathrm{M}(c)$ is given by (41).

When the coils have the same length

$$
\mathbf{M}=\mathbf{M}(h-2 l)+\mathrm{M}(h+2 l)-2 \mathrm{M}(h) . .
$$


Self and Mutual Induction of Coavial Coils.

(b) Overlapping coils.

Regard the ficld inside the outer coil as made up of two portions,

(1) the uniform field calculated by assuming the coil to be part of an infinite coil ;

(2) the field due to the polarity of the coil faces.

If $\mathrm{MI}_{1}, \mathrm{M}_{2}$ are the linkages through the second coil due to these two fields, then $\mathrm{M}=\mathrm{M}_{1}+\mathrm{M}_{2} ; \mathrm{M}_{2}$ is given by (42) and (41) (no regard being paid to the sign of $c$ ), and

$$
\mathrm{M}_{1}=\frac{4}{3} \pi^{2} n_{1} n_{9} l_{i}\left(a_{2}-a_{1}\right)\left(b_{2}^{3}-b_{1}^{3}\right), \quad . \quad .
$$

$k$ being the length of the overlap.

When the coils have common centres, $l=0$, and (42) becomes

$$
\mathrm{M}_{2}=2\left\{\mathrm{M}\left(l_{1}+l_{2}\right)-\mathrm{M}\left(l_{1}-l_{2}\right)\right\} \ldots .
$$

If in addition they have the same length (2l),

$$
\mathrm{M}_{2}=2\{\mathrm{M}(2 l)-\mathrm{M}(0)\}
$$

11. For the purposes of calculation, it is convenient to alter the notation as in the following example.

Let the coils have the following dimensions :-

Outer radii ..... $a_{3}=10 \mathrm{~cm} ., b_{2}=4 \mathrm{~cm}$.

Inner radii $\ldots . . . \quad a_{1}=5 \mathrm{~cm} ., b_{1}=2 \mathrm{~cm}$.

Lengths $\ldots \ldots \ldots .2 l_{1}=6 \mathrm{~cm} ., 2 l_{2}=44 \mathrm{~cm}$.

Displacement of centres $=h=21 \mathrm{~cm}$.

Then since the amount of overlapping is $4 \mathrm{~cm}$, we have from (43)

$$
\frac{\mathrm{M}_{1}}{2 \pi^{2} n_{1} n_{2}}=\frac{2}{3} \times 4 \times 5 \times\left(4^{3}-2^{3}\right)=746 \cdot 7 .
$$

Again,

$$
\begin{array}{ccc}
h-l_{1}-l_{2}=c_{1}=4, & c_{1} / a_{2}=z_{1}=0 \cdot 4, & c_{1} / a_{1}=z_{1}{ }^{\prime}=0 \cdot 8 \\
h+l_{1}+l_{2}=c_{2}=46, & c_{2} / a_{2}=z_{2}=4 \cdot 6, & c_{2} / a_{1}-z_{2}{ }^{\prime}=9 \cdot 2 \\
h-l_{1}+l_{2}=c_{3}=40, & c_{3} / a_{2}=z_{3}=4 \cdot 0, & c_{3} / a_{1}=z_{3}{ }^{\prime}=8 \cdot 0 \\
h+l_{1}-l_{2}=c_{4}=2, & c_{4} / a_{2}=z_{4}=0 \cdot 2, & c_{4} / a_{1}=z_{4}{ }^{\prime}=0 \cdot 4 \\
b_{2} / a_{2}=r_{1}=0 \cdot 4, b_{1} / a^{2}=r_{2}=0 \cdot 2, b_{2} / a_{1}=r_{1}=0.8, h_{1} / a_{1}=r_{2}{ }^{\prime}=0 \cdot 4 . \\
\text { * Maxwell, ' Electricity and Magnetism,' vol. ii. p. 312. }
\end{array}
$$


Therefore by (41) and (42), using the notation $\xi$ of the Tables and putting

$$
\begin{aligned}
& \xi_{p q} \text { for } \xi\left(z_{p}, r_{q}\right), \quad \xi_{p q}^{\prime} \text { for } \xi\left(z_{p}{ }^{\prime}, r_{q}{ }^{\prime}\right) \\
& \frac{\mathbf{M}_{2}}{2 \pi^{2} n_{1} n_{2}}=a_{2}^{5}\left\{r_{1}^{3}\left(\xi_{11}+\xi_{21}-\xi_{31}-\xi_{41}\right)-r_{2}^{3}\left(\xi_{12}+\xi_{22}-\xi_{32}-\xi_{12}\right)\right\} \\
& -a_{1}^{5}\left\{r_{1}^{\prime}{ }^{3}\left(\xi_{11}^{\prime}+\xi_{21}^{\prime}-\xi_{31}^{\prime}-\xi_{41}^{\prime}\right)-r_{2}^{\prime}{ }_{2}^{3}\left(\xi_{12}^{\prime}+\xi_{22}^{\prime}-\xi_{32}^{\prime}-\xi_{42}^{\prime}\right)\right. \\
& =10^{5}\left(-\frac{4^{3}}{10^{3}} \times 0.02820+\frac{2^{3}}{10^{3}} \times 0 \cdot 02978\right) \\
& -5^{5}\left(-\frac{8^{3}}{10^{3}} \times 0.036 .52+\frac{4^{3}}{10^{3}} \times 0.03077\right) \\
& =-104 \cdot 4 \text {. } \\
& \therefore \mathrm{M}=\mathrm{M}_{1}+\mathrm{M}_{2}=2 \pi^{2} n_{1} n_{2}(746 \cdot 7-104 \cdot 4)=12,680 n_{1} n_{2} \text {. }
\end{aligned}
$$

\section{Self-inductances.}

Since the self-induction of a coil is the same as the matual induction between two coincident coils, we have by the method of section 10 ,

$$
\mathrm{L}=\mathrm{L}_{1}+\mathrm{L}_{2}
$$

in which $\mathrm{L}_{1}$ is the self-induction calculated by assuming the coil to be part of an infinite coil and $\mathrm{L}_{2}$ are the linkages due to the polar field of the coil.

If the coil has length $c$, outer radius $a$, inner radius $b$, winding density $n$,

$$
\mathrm{L}_{1}=\frac{2}{3} \pi^{2} n^{2} a^{5} z(1-r)^{2}\left(1+2 r+3 r^{2}\right), \quad . \quad .
$$

in which $z=c / a, r=b / a$, and no allowance is made for the space occupied by insulation.

Also $\mathrm{L}_{2}$ is given by the $\mathrm{M}_{2}$ of $(42 c)$ in which $(41 a)$ holds, so that, using the present notation,

$$
\left.\begin{array}{c}
\mathrm{L}_{2}=2\{\mathrm{M}(c)-\mathrm{M}(0)\} . . . \\
\left.\mathrm{M}(c) / n^{2}=a^{\tilde{s}}\left\{\mathrm{~N}(z, 1)-2 \mathrm{~N}(z, r)+r^{3} \mathrm{~N}\left(\frac{z}{r}, 1\right)\right\}\right\}, \\
\mathrm{M}(0) / n^{2}=a^{j}\left\{\left(1+r^{3}\right) \mathrm{N}(0,1)-2 \mathrm{~N}(0, r)\right\}
\end{array}\right\},
$$

or using the notation $\xi, \eta$ of the tables,

$$
\begin{aligned}
& \mathrm{M}(c) / 2 \pi^{2} n^{2} a^{5}=\xi(z, 1)-2 r^{3} \xi(z, r)+r^{5} \xi\left(\frac{z}{r}, 1\right) \\
& \quad \text { when } z<4, \\
& \left.\mathrm{M}(c) z / \pi^{2} n^{2} a^{5}=\frac{1}{9}\left(1-r^{3}\right)^{2}-\eta(z, 1)+2 r^{3} \eta\left(z, r^{\prime}\right)-r^{6} \eta\left(\frac{z}{r}, 1\right)\right\} \text {. } \\
& \quad \text { when } z>4 \\
& \quad \text { and } \\
& \mathrm{M}(0) / 2 \pi^{2} n^{2} a^{5}=\left(1+r^{s}\right) \xi(0,1)-2 r^{3} \xi(0, r) .
\end{aligned}
$$


Self and Mutual Tnduction of Coavial Coils.

When $z>4$, we obtain an accuracy of 1 in 10,000 if we use

$$
\mathrm{L}=\mathrm{L}_{1}\left(1-\frac{\alpha}{z}+\frac{\beta}{z^{2}}-\frac{\gamma}{z^{4}}\right), . . . .
$$

in which $\alpha, \beta, \gamma$ are functions of $r$ and are tabulated below.

TABLE V.

\begin{tabular}{|c|c|c|c|}
\hline$r$ & a. & $\beta$. & $\gamma$ \\
\hline $\begin{array}{l}0.0 \\
0.2 \\
0.4 \\
0.6 \\
0.8 \\
1.0\end{array}$ & $\begin{array}{l}0.73238 \\
0.73699 \\
0.75574 \\
0.78447 \\
0.81718 \\
0.84883\end{array}$ & $\begin{array}{c}0.33333 \\
0.33719 \\
0.35579 \\
0.39042 \\
0.43906 \\
0.50000\end{array}$ & $\begin{array}{l}0 \cdot 0953 \\
0 \cdot 0973 \\
0 \cdot 1071 \\
0 \cdot 1306 \\
0 \cdot 1701 \\
0 \cdot 2306\end{array}$ \\
\hline
\end{tabular}

The 'values of $\alpha, \beta, \gamma$ have been calculated from the formulæ

$$
\begin{aligned}
q \alpha & =2\left\{\left(1+r^{5}\right) \xi(0,1)-2 r^{3} \xi(0, r),\right. \\
q \beta & =\frac{1}{9}\left(1-r^{3}\right)^{2}, \\
q \gamma & =z^{2}\left\{\eta(z, 1)-2 r^{3} \eta(z, r)+r^{6} \eta\left(\frac{z}{r^{2}}, 1\right)\right\}, \\
q & =\frac{1}{3}(1-r)^{2}\left(1+2 r+3 r^{2}\right),
\end{aligned} .
$$

with $z=4$ in the expression for $\gamma$. Hence the tabulated values of $\gamma$ are only correct for $z=4$. However, for larger values of $z$, the error in the final result is always less than 1 in 10,000 .

The above formulæ fail when $r=1$, but in this case the coil becomes a thin cylinder, the self-induction of which is

$$
\mathrm{L}=\mathrm{L}_{1}\left(1-\frac{8}{3 \pi} \frac{1}{z}+\frac{1}{2} \frac{1}{z^{2}}-\frac{1}{4} \frac{1}{z^{4}}+\frac{5}{16} \frac{1}{z^{6}}-\frac{35}{64 z^{8}}+\ldots\right) \cdot(50)^{*}
$$

so that for $r=1$,

$$
\alpha=\frac{8}{3 \pi}, \quad \beta=\frac{1}{2}, \quad \gamma=\frac{1}{4}-\frac{5}{16 z^{2}}+\frac{35}{64 z^{4}}-\ldots
$$

* Russell. Phil. Mag. vol. xiii. p. 420 (1907); IIavel̀ock, Phil. Mag. vol. xv. p. 332 (1908). 
13. In order to illustrate the method of working for short coils, take a coil having

$$
\begin{aligned}
& \text { outer radius }=a=4 \mathrm{~cm} \text {., } \\
& \text { inner radius }=b=2 \mathrm{~cm} \text {., } \\
& \text { length }=c=4 \mathrm{~cm} \text {. } \\
& \text { Then } \quad z=c / a=l, \quad r=b / a=0.5 \text {. } \\
& \text { By (44) } \quad \mathrm{L}_{1} / 2 \pi^{2} n^{2} a^{5}=0 \cdot 229167 \text {. } \\
& \text { By }(47 a) \\
& \begin{aligned}
\mathrm{M}(c) / 2 \pi^{2} n^{2} a^{5} & =\xi(1,1)-2(0.5)^{3} \xi(1,0.5)+(0.5)^{5} \xi(2,1), \\
& =0.045477-\frac{1}{4} \times 0.048216+\frac{1}{32} \times 0.026022, \\
& =0.034236,
\end{aligned} \\
& \mathrm{M}(0) / 2 \pi^{2} x^{2} a^{5}=\left(1+\frac{1}{32}\right) \times 0 \cdot 122062-\frac{1}{4} \times 0 \cdot 150930, \\
& =0.088144 \text {. } \\
& \text { Therefore } \quad \mathrm{L}=\mathrm{L}_{1}-2 \mathrm{M}(0)+2 \mathrm{M}(c) \\
& =2 \pi^{2} n^{2} a^{5}(0.121351) \\
& =2453 \cdot 9 n^{2} \text {. } \\
& \mathrm{L}=2459 \cdot 5 n^{2} \text {, }
\end{aligned}
$$

so that the error in using the latter formula for this coil is $0 \cdot 23$ per cent.

\section{Conclusion.}

In applying the formulæ and tables, their range of application should be borne in mind. They are intended to be used only when the inner and outer diameters of the coils differ appreciably $(b / a<0.8)$, and when the coil-lengths are not too small $(c / a>0 \cdot 2)$. An exception to this rule is Table V. which (with graphical interpolation) holds for all values of $b / a$. For coils whose dimensions are outside these limits the usual solenoid or circular filament formuiæ are more suitable, the geometric mean distance correction being applied to the channel section.

It should also be noted that no allowance is made for the insulation space of the winding.

Finally, by successive differentiation of the formulæ for the function $\mathrm{N}$, many known formulæ for the mutual induction between solenoids, flat coils, and circles may be obtained.

* Fleming, 'Principles of Electric Wave Telegraphy', p. 140 (2nd edition; , 